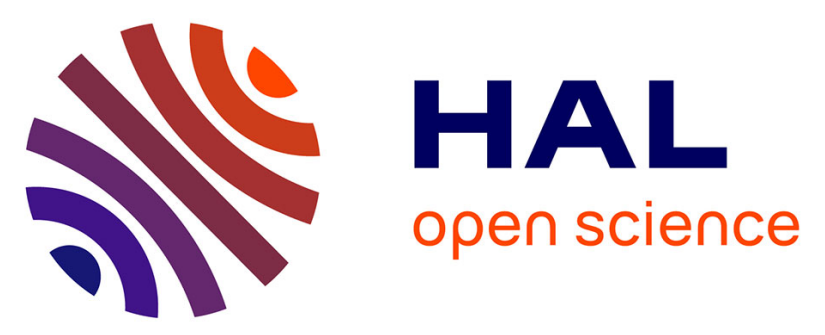

\title{
On the use of optimization methods for the minimization of fertilizer application error with centrifugal spreaders
}

Teddy Virin, Jonas Koko, Emmanuel Piron, Philippe Martinet

\section{To cite this version:}

Teddy Virin, Jonas Koko, Emmanuel Piron, Philippe Martinet. On the use of optimization methods for the minimization of fertilizer application error with centrifugal spreaders. ICINCO06 - 3rd International Conference on Informatics in Control, Automation and Robotics, Aug 2006, Setubal, Portugal. hal-02468330

\section{HAL Id: hal-02468330 \\ https://inria.hal.science/hal-02468330}

Submitted on 5 Feb 2020

HAL is a multi-disciplinary open access archive for the deposit and dissemination of scientific research documents, whether they are published or not. The documents may come from teaching and research institutions in France or abroad, or from public or private research centers.
L'archive ouverte pluridisciplinaire HAL, est destinée au dépôt et à la diffusion de documents scientifiques de niveau recherche, publiés ou non, émanant des établissements d'enseignement et de recherche français ou étrangers, des laboratoires publics ou privés. 


\title{
ON THE USE OF OPTIMIZATION METHODS FOR THE MINIMIZATION OF FERTILIZER APPLICATION ERROR WITH CENTRIFUGAL SPREADERS
}

\author{
Teddy Virin \\ Cemagref \\ 03150 Montoldre, France \\ Email: teddy.virin@cemagref.fr \\ Jonas Koko \\ LIMOS, Université de Blaise Pascal \\ 63177 Aubière cedex, France \\ Email: koko@isima.fr \\ Emmanuel Piron \\ Cemagref \\ 03150 Montoldre, France \\ Email: emmanuel.piron@cemagref.fr \\ Philippe Martinet \\ LASMEA, Université de Blaise Pascal \\ 63177 Aubière cedex, France \\ Email: Philippe.MARTINET@lasmea.univ-bpclermont.fr
}

Keywords: Optimization, Augmented lagrangian, Centrifugal spreading, Fertilizer application error.

\begin{abstract}
Fertilizer application is one of the most important operations in agricultural production. Thanks to their low cost and robustness, centrifugal spreaders are widely used to carry out this task. However, when distances between successive paths followed by the tractor in the field are not constant, application errors occur. These ones generally cause waters pollution and economic issues. In this paper, to limit harmful environmental effects and disastrous drop in production due to centrifugal spreading, we propose an approach based on optimization techniques to improve the fertilization quality. An optimization criterion relying on a spatial distribution model, obtained in previous works, is considered. To compute optimal parameters which should be used as reference variables for the control of the spreader in the future, mechanical constraints are introduced. Faced with a large scale problem, we use an augmented lagrangian algorithm combined with a l-bfgs technique. Simulations results show low application error values comparing to fertilization inaccuracies found without optimization.
\end{abstract}

\section{INTRODUCTION}

Fertilization operation is commonly achieved to apply nutrients to make up the soil deficiencies and therefore enable a correct growth of plants in farmlands. Centrifugal spreading is the main technique used to distribute mineral fertilizers with respect to desired doses calculated from agronomical and pedological reasonings. This method permits to have uniform distributions as long as the trajectories followed by the tractor equipped with spreader are parallel and regularly spaced. Unfortunately, the use of this kind of applicator is quickly limited when geometrical singularities, such as irregularly spaced tramlines, non parallel paths or start and end of spreading, occur. Indeed, at these spots, local application errors can be observed. In some cases, over-application can lead to an eutrophication phenomenon causing the disappearance of aquatic species (Isherwood, 1998). Moreover, when the distributed amounts are below the prescribed dose, the final production is often very low. Thus, these statements lead the different governments in Northern Europe to impose some strict rules as specified in (Bruxelles, 2005). Confronted with these requirements, many researches are carried out in order to not only reduce dramatic environmental effects but also increase margins in agricultural crop production. In this study, we focus on a new approach based on optimization techniques. We consider a cost function formalized from the actual spatial distribution model instead of using the traditional method relying on the best arrangement of transverse distribution. To 
take into account the mechanical limits of applicator, constraints are also considered. Thus, the computed optimal parameters should be used as reference values for future works dealing with the control of spreader. This paper is organized as follows. The next section exposes the centrifugal spreading principles and the related drawbacks. In section 3 , the cost function modeling is dealt with. To solve the problem in an efficient way, the problem decomposition and the optimization techniques are detailed in section 4 . In the last section, because of more complicated phenomena occurring in boundary zones, we apply this method along parallel and non parallel trajectories only in the main field body and expose simulation results.

\section{CENTRIFUGAL SPREADING DRAWBACKS}

According to the soil and crops characteristics, agricultural engineers state the amounts to be applied within the farmland. These desired doses often takes the form of prescribed dose map. This map depicts the field which is virtually gridded and where each mesh corresponds to the previously specified doses. Fertilization aims to distribute nutrients so that the actual spread doses get close to the prescribed ones. This spreading regularity is above all performed by centrifugal spreaders with double spinning discs which eject fertilizers along each tramline in the field. The functioning principles of these applicators are very simple. Indeed, while the tractor progresses, fertilizers granulars contained in the hopper pour onto each disc and are ejected by centrifugal effect.

Nowadays, with precision farming technologies, in order to apply inputs according to the machine location and a prescribed dose map, tractor-implement combination is equipped with a GPS antenna, a radar speed sensor and an actuator. The two first tools enable to know the tractor position and speed. Concerning the actuator, it permits to control the fertilizer mass flow rate. The actual amount of applied fertilizers currently called spread pattern has an irregular distribution which is often underlined by the transverse distribution curve obtained by summing the amounts along each travel direction. An example of spread pattern and its related transverse distribution for only one disc are depicted in Figure 1. Thus, this spatial heteregeousness lead the tractor driver to follow outward and return paths in order to have an uniform deposit from transverse distribution summation for each successive tramlines within the arable land. This strategy is detailed in Figure 2. As we can notice, fertilization strategy is essentially based on the best overlappings of transverse distribution according to the different tractor trajectories. This method is applied for

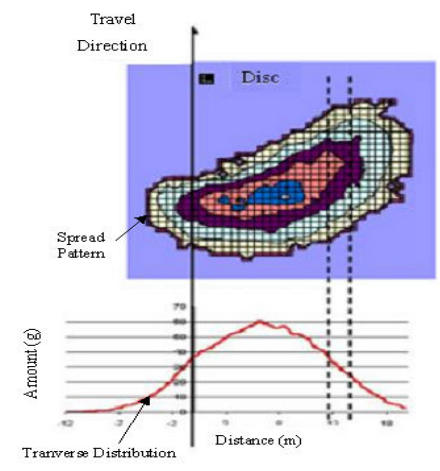

Figure 1: Spread pattern (spatial distribution) and transverse distribution (red curve)

experiments undertaken to evaluate fertilizer application accuracy or to investigate device settings according to tests procedures such as (ISO, 1985). So, these procedures enable to select optimal machine settings in order to obtain a regular deposit. Some studies relying on these tests are also led to assess performance of applicators like in (Yule et al., 2005). Deposits are considered to be uniform when the distance between two overlapping lines are equal to the distance separating two successive tramlines. Moreover, if this condition is checked, overlapping lines are symmetry axes which make two consecutive paths coincide. In this case, the distance between two successive trajectories, called working width, is said optimal. Unfortunately, when applying this strategy in the field, the actual phenomenon occurring during spreading process is ignored. Indeed, the deposit on the ground results in fact from the accumulation of several amounts distributed for different spreader GPS positions. Therefore, when geometrical singularities occur, like non parallel paths or start and end of spreading, fertilization application errors appear as illustrated in Figure
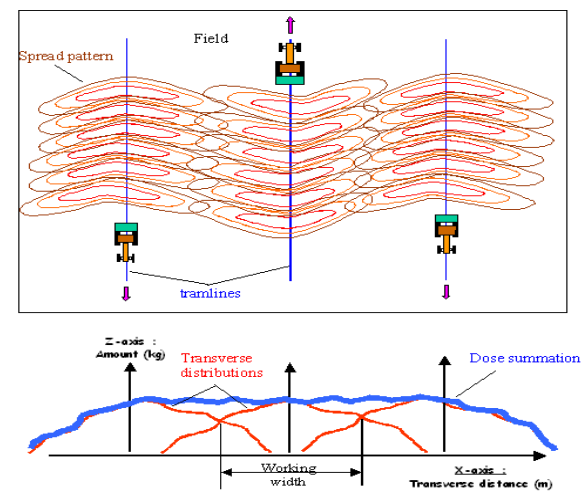

Figure 2: Fertilization strategy based upon transverse distribution summation. 


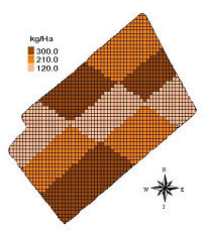

(a) Prescribed dose map obtained from agronomical considerations.

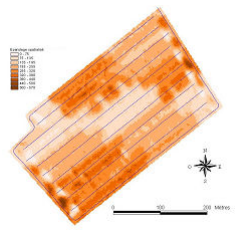

(b) Actual dose map obtained by applying the fertilization method based on adjustment of transverse distribution.

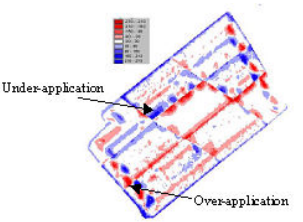

(c) Application errors map calculated from the difference between the prescribed dose map and actual dose map.

Figure 3: Application errors resulting from the reasoning based on the best transverse distribution investigation and not on spread patterns overlappings.

3(c) where results are obtained by simulating spread patterns overlappings with settings imposed by manufacturers rules. To limit these issues, a solution could be to look for optimal paths for the machine as in (Dillon et al., 2003). However, this kind of solution cannot be applied when tramlines are already fixed by other agricultural operations like sowing. So, it is clear that some efforts must be done to achieve better spread patterns arrangement according to geometrical constraints met in farmland during fertilization practice. The computed adjustments should be continuously achieved for each position of applicator by modifying its settings. In this work, we study then a method which permits to calculate optimal parameters to have the best spread patterns arrangement within the field in the presence of imposed tramlines.

\section{COST FUNCTION}

In order to develop a suitable optimization criterion, we must consider the spread pattern model. This model needs then the definition of some parameters such as the time $(t \in \mathbb{R})$, the spatial domain, in other words the field, $\left(\Omega \in \mathbb{R}^{2}\right)$, the path $(s(t) \in \Omega)$, the coordinates of points $(x \in \Omega)$, the distance between $s(t)$ and $x(r(x, t) \in \mathbb{R})$ and the angle between $\overrightarrow{s(t) x}$ and $s(t)(\theta(x, t) \in \mathbb{R})$. The spread pattern shown in Figure 1 , is currently defined by its medium radius and medium angle. The first parameter, varying with the speed of disc, corresponds to the distance between the disc centre and the spread pattern one while the second, modifiable with the fertilizers dropping point on the disc, states the angle between the travel direction and the straight line passing through the disc centre and the spatial distribution one. Here, the respective mass flow rates for the right and left discs are defined by $m(t)$ and $d(t) . \rho(t)$ and $\xi(t)$ stand for the medium radius related to the right and left discs respectively. At last, $\xi(t)$ and $\psi(t)$ correspond to the right and left discs medium angles. All these parameters are defined in $\mathbb{R}$. By assuming $\sigma_{r}$ and $\sigma_{\theta}$ to be the respective constant standard deviations for the medium radius and the medium angle, we can calculate the right and left spatial distributions $q_{r}$ and $q_{l}$, according to (Olieslagers, 1997), as:

$$
\begin{aligned}
& q_{r}(x, m(t), \rho(t), \varphi(t))=\tau \cdot \exp \left(-A(x, t)^{2} / a\right) \\
& \cdot \exp \left(-B(x, t)^{2} / b\right) \\
& q_{l}(x, d(t), \xi(t), \psi(t))=\kappa \cdot \exp \left(-C(x, t)^{2} / a\right) \\
& \cdot \exp \left(-D(x, t)^{2} / b\right) \\
& \text { with } \quad A(x, t)=r(x, t)-\rho(t) \\
& B(x, t)=\theta(x, t)-\varphi(t) \\
& C(x, t)=r(x, t)-\xi(t) \\
& D(x, t)=\theta(x, t)-\psi(t)
\end{aligned}
$$

and where $a=2 \sigma_{r}^{2}, b=2 \sigma_{\theta}^{2}, \tau=m(t) /\left(2 \pi \sigma_{r} \sigma_{\theta}\right)$ and $\kappa=d(t) /\left(2 \pi \sigma_{r} \sigma_{\theta}\right)$. To simplify notations, we define $M(t)=(m(t), d(t)) \in \mathbb{R}^{2}, R(t)=$ $(\rho(t), \xi(t)) \in \mathbb{R}^{2}$ and $\Phi(t)=(\varphi(t), \psi(t)) \in \mathbb{R}^{2}$. The global distribution is then obtained as the summation of right and left contributions:

$$
\begin{aligned}
q_{\text {tot }}(x, M(t), R(t), \Phi(t)) & =q_{r}(x, m(t), \rho(t), \varphi(t)) \\
& +q_{l}(x, d(t), \xi(t), \psi(t))(7)
\end{aligned}
$$

Thus, the actual distributed dose $Q \in \mathbb{R}^{2}$ during the interval of time $(0, T)$ for single tramline can be calculated as:

$$
Q(x, M, R, \Phi)=\int_{0}^{T} q_{t o t}(x, M(t), R(t), \Phi(t)) d t
$$

If $Q^{*}$ stands for the prescribed dose, the cost function to be minimized is given by:

$$
F(M, R, \Phi)=\int_{\Omega}\left[Q(x, M, R, \Phi)-Q^{*}\right]^{2} d x
$$


Given that (9) cannot be calculated in an analytical way, discretization is necessary. So, $\Omega$ is gridded so that $Q$ and $Q^{*}$ can be computed with bilinear approximations. A temporal discretization is also carried out. This is done by dividing the interval $(0, T)$ into $n$ elements with equal length $\delta=T / n$. We can then define $t_{j}=j \delta$ with $j=0,1, \ldots, n$. Consequently, we can assume $M_{j}=M\left(t_{j}\right), R_{j}=R\left(t_{j}\right)$ and $\Phi_{j}=\Phi\left(t_{j}\right)$. The corresponding vectors are defined as $\boldsymbol{M}=\left[M_{0} \cdots M_{n}\right]^{T}, \boldsymbol{R}=\left[R_{0} \cdots R_{n}\right]^{T}$ and $\boldsymbol{\Phi}=\left[\Phi_{0} \cdots \Phi_{n}\right]^{T}$. In order not to untimely solicit actuators, constraints are introduced. So, the functions $M, R$ and $\Phi$ and their time derivative are subject to bound constraints. The set of solutions is then defined by $S=\left\{(\boldsymbol{M}, \boldsymbol{R}, \boldsymbol{\Phi}) \in \mathbb{R}^{6(n+1)}\right\}$ so that:

$$
\left\{\begin{array}{l}
M_{\min } \leq \boldsymbol{M} \leq M_{\max } \\
R_{\min } \leq \boldsymbol{R} \leq R_{\max } \\
\Phi_{\min } \leq \boldsymbol{\Phi} \leq \Phi_{\max } \\
\left|M_{i+1}-M_{i}\right| \leq \alpha \delta \\
\left|R_{i+1}-R_{i}\right| \leq \beta \delta \\
\left|\Phi_{i+1}-\Phi_{i}\right| \leq \gamma \delta,
\end{array}\right.
$$

where $\alpha, \beta$ and $\gamma$ are known parameters fixed with respect to the machine mechanical characteristics. We obtain then a nonlinear programming problem given by:

$$
\min _{(\boldsymbol{M}, \boldsymbol{R}, \boldsymbol{\Phi}) \in S} F(\boldsymbol{M}, \boldsymbol{R}, \boldsymbol{\Phi})
$$

The set $S$ is in our case bounded closed. Thus, according to the Weierstrass theorem, the problem $(\mathcal{P})$ has at least one solution. In most cases, there exist several tramlines and the actual distributed dose for all trajectories is then obtained by the summation of the applied dose for each indexed $\mathrm{k}$ path:

$$
\begin{aligned}
Q(x, U) & =\sum_{k=1}^{w} Q_{k}(x, U) \\
\text { with } Q_{k}(x, U) & =\int_{t_{i}^{k}}^{t_{f}^{k}} q_{t o t}(x, M(t), R(t), \Phi(t)) d t
\end{aligned}
$$

and where $U=(M, R, \Phi)$. Here, $w$ is the number of paths and the trajectories $s^{k}(t)$ are assumed to be defined in the interval $\left(t_{i}^{k}, t_{f}^{k}\right)$. If we consider also the definitions of $M_{j}^{k}=M\left(t_{j}^{k}\right), R_{j}^{k}=R\left(t_{j}^{k}\right)$, and $\Phi_{j}^{k}=\Phi\left(t_{j}^{k}\right)$ we can then use the discretization techniques as before. Consequently, from these definitions, optimization in the whole field considering all paths can be carried out by solving the problem $(\mathcal{P})$.

\section{METHODOLOGY OF OPTIMIZATION}

Fields mostly include many tramlines several hundred metres long. If we apply the discretization scheme previously detailed, we are confronted with a large scale problem. Indeed, in our case, like most prescribed dose map, the field is $1 \mathrm{~m}$ gridded. To lose informations as little as possible, 2 samples of parameters per elementary mesh are computed. So if we consider for example only 4 tramlines $100 \mathrm{~m}$ long within the farmland, after discretization, the number variables raises to 4800 . From this statement, it is clear that solving the optimization problem without decomposition is prohibitive. Let us precise some notations before the decomposition explanations:

$$
\begin{gathered}
K_{1}=\{k \in \mathbb{N} \mid 1 \leq k \leq w\}, \\
K_{2}=\{k \in \mathbb{N} \mid 1 \leq k \leq w-1\}, \\
K_{3}=\{k \in \mathbb{N} \mid 2 \leq k \leq w\}, \\
L_{1}=\{l \in \mathbb{N} \mid \forall z \geq 2 \in \mathbb{N}, 1 \leq l \leq z\}, \\
L_{2}=\{l \in \mathbb{N} \mid \forall z \geq 2 \in \mathbb{N}, 2 \leq l \leq z\}, \\
\Omega=\bigcup_{k \in K_{1}} \Omega^{k}, \quad \Omega^{k}=\bigcup_{l \in L_{1}} \Omega_{l}^{k},
\end{gathered}
$$

with $\Omega^{k} \in \mathbb{R}^{2}$ the $k^{\text {th }}$ subdomain of $\Omega$, and $\Omega_{l}^{k} \in \mathbb{R}^{2}$ the $l^{\text {th }}$ subdomain of $\Omega^{k}$. Here, we decompose the problem so that each path $s^{k}(t)$ is individually dealt with. So, the subdomains $\Omega^{k}$ are defined so that:

$$
\begin{aligned}
& \partial \Omega^{k} \cap \Omega^{k+1}=s^{k+1}(t), \forall(k, t) \in K_{2} \times\left(t_{i}^{k+1}, t_{f}^{k+1}\right) \\
& \partial \Omega^{k} \cap \Omega^{k-1}=s^{k-1}(t), \forall(k, t) \in K_{3} \times\left(t_{i}^{k-1}, t_{f}^{k-1}\right)
\end{aligned}
$$

In order to make easier to understand the spatial decomposition, Figure 4 illustrates the example of three parallel tramlines in a domain $\Omega$ with a rectangular geometry.

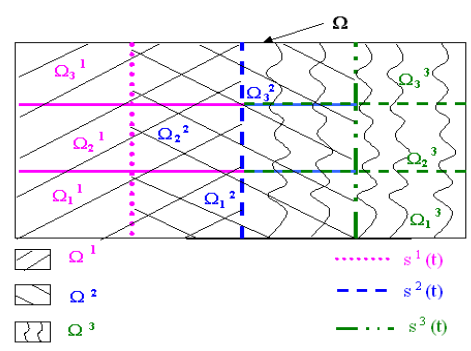

Figure 4: Rectangular domain $\Omega$ divided into 9 subdomains $\Omega_{l}^{k}, 1 \leq l \leq 3,1 \leq k \leq 3$.

Moreover, if we assume the vectors $\boldsymbol{M}_{l}^{k}, \boldsymbol{R}_{l}^{k}, \boldsymbol{\Phi}_{l}^{k}$ to be the respective restrictions of $M, R$ and $\Phi$ in the subdomain $\Omega_{l}^{k}$, we can also define also the set $S_{l}^{k}$ as the restriction of $S$ in the same subdomain. By taking into account the symmetries conditions exposed in section 2, the natural decomposition of $(\mathcal{P})$ is given by:

$\left(\mathcal{P}^{\prime}\right)\left\{\begin{array}{l}\min \sum_{l=1}^{z} \sum_{k=1}^{w} J_{l}^{k}\left(x, \boldsymbol{M}_{l}^{k}, \boldsymbol{R}_{l}^{k}, \boldsymbol{\Phi}_{l}^{k}\right) \\ \left(\boldsymbol{m}_{l}^{k}, \boldsymbol{R}_{l}^{k}, \boldsymbol{\Phi}_{l}^{k}\right) \in S_{l}^{k},(l, k) \in L_{1} \times K_{1}\end{array}\right.$

where

$$
J_{l}^{k}\left(x, \boldsymbol{M}_{l}^{k}, \boldsymbol{R}_{l}^{k}, \boldsymbol{\Phi}_{l}^{k}\right)=\int_{\Omega_{l}^{k}}\left[Q_{l}^{k}(x)-Q^{*}\right]^{2} d x
$$


with $Q_{l}^{k}(x)$ the actual distributed dose within $\Omega_{l}^{k}$ taking into account not only the amounts already applied in $\Omega_{l}^{k-1}$ and $\Omega_{l-1}^{k}$ but also the future distributed dose along the path $s^{k+1}(t)$ which is predicted so that it respects the symmetries properties previously explained. The problem $\left(\mathcal{P}^{\prime}\right)$ is an optimization problem subject to inequality constraints. For $(l, k) \in L_{1} \times K_{1}$, to minimize the functional $J_{l}^{k}$ we consider then the problem $\left(\mathcal{P}_{\text {ineq }}\right)$ defined as:

$$
\left(\mathcal{P}_{\text {ineq }}\right) \quad\left\{\begin{array}{l}
\min J_{l}^{k}\left(\boldsymbol{M}_{l}^{k}, \boldsymbol{R}_{l}^{k}, \boldsymbol{\Phi}_{l}^{k}\right) \\
u_{j} \leq h_{j}\left(\boldsymbol{M}_{l}^{k}, \boldsymbol{R}_{l}^{k}, \boldsymbol{\Phi}_{l}^{k}\right) \leq v_{j}, \\
j=1,2, \ldots \operatorname{dim}\left(\boldsymbol{M}_{l}^{k}\right)
\end{array}\right.
$$

where $h_{j}$ denotes the $j^{\text {th }}$ double inequality, $u_{j}$ and $v_{j}$ its lower and upper bound. In order to obtain an acceptable solution after algorithm execution and avoid solving the problem which consists in determining saturated constraints, we choose to apply an augmented lagrangian algorithm (Bertsekas, 1982) associated with a l-bfgs technique shown to be efficient with large scale optimization problem (Byrd et al., 1994). Moreover, desirous of obtaining the best fertilizer application in the field, it is important to note that the optimization algorithm is not time-bounded.

\section{APPLICATION}

Here, we focus essentially on main fiel body application and not on boundaries area where more complex phenomena occur. A constant prescribed dose fixed at $100 \mathrm{Kg} / \mathrm{Ha}$ is considered because even in the case of uniform desired rate, application errors appear. The speed of tractor is also constant and equal to $10 \mathrm{Km} / \mathrm{h}$. The studied farmland is illustrated in Figure 5.

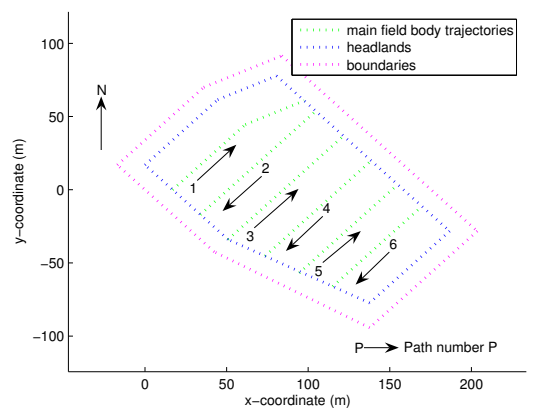

Figure 5: Field with parallel and non parallel tramlines

The default working width is fixed at $24 \mathrm{~m}$. A narrowing occurs at the end of the first path. The distance between the $4^{\text {th }}$ and $5^{\text {th }}$ tramlines is $23 \mathrm{~m}$ while it is equal to $21 \mathrm{~m}$ between the $5^{\text {th }}$ and $6^{\text {th }}$ trajectories. In practice, the manufacturers settings do not vary during time and are determined to be optimal with $24 \mathrm{~m}$ working width. With these settings, we obtain errors shown in Figure 6. As we can notice, over-application areas appear on the left and the right of the $4^{\text {th }}$ tram-

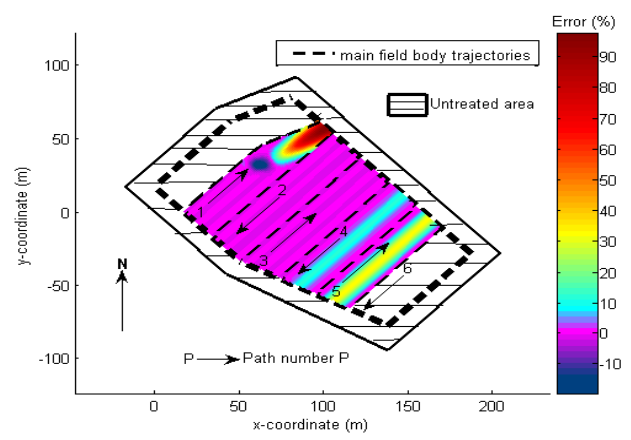

Figure 6: Application errors obtained with the manufacturers settings

line. Over-dosage is very important at the end of the first path and almost reaches $95 \%$. Futhermore, we can distinguish an under-application zone slightly below the break-point which marks the travel direction change for this same trajectory. Everywhere else, error is included between $-6 \%$ and $+7 \%$ and is then acceptable. To reduce these fertilization errors, we apply our optimization methodology as detailed in the previous section making sure that the considered mechanical constraints gather the characteristics of the most used spreader. As in practice, for consecutive parallel tramlines, optimal parameters are computed so that they are time independent. So, calculated variables for the paths 3 to 6 are constant during time and are recapitulated in Table 1. These values are very close to actual current values.

Table 1: Optimal values for successive parallel tramlines $\left(\mathrm{M}_{\mathrm{f}}\right.$ : Mass Flow Rate $(\mathrm{Kg} / \mathrm{min}) ; \mathrm{R}_{\mathrm{m}}$ : Medium Radius; $\theta_{\mathrm{m}}$ : Medium Angle $\left({ }^{\circ}\right)$ )

\begin{tabular}{|l|c|c|c|c|}
\hline \multicolumn{5}{|c|}{ Left Disc } \\
\hline & Path 3 & Path 4 & Path 5 & Path 6 \\
\hline $\mathbf{M}_{\mathbf{f}}$ & 19.32 & 17.92 & 19.41 & 20.6 \\
\hline $\mathbf{R}_{\mathbf{m}}$ & 15.13 & 14.61 & 14.7 & 15.17 \\
\hline$\theta_{\mathbf{m}}$ & -20.78 & -20.96 & -19.66 & -19.79 \\
\hline \multicolumn{5}{|c|}{ Right Disc } \\
\hline & Path 3 & Path 4 & Path 5 & Path 6 \\
\hline $\mathbf{M}_{\mathbf{f}}$ & 20.7 & 21.24 & 17.26 & 16.95 \\
\hline $\mathbf{R}_{\mathbf{m}}$ & 15.49 & 15.55 & 14 & 14 \\
\hline$\theta_{\mathbf{m}}$ & 18.93 & 18.37 & 18.61 & 18.79 \\
\hline
\end{tabular}

Unlike the previous paths, optimal variables for the two first trajectories are dependent time. The optimized parameters for the first path are shown in Figure 7. The parameters for the left and right discs appear respectively on the left and the right. As expected, after the travel direction change, the medium radius drops. For the left disc, the mass flow rate 
slightly varies around break-point and seems not to be affected by the occurring narrowing. However, for the right disc, it increases before the break-point and drops after this one. Concerning the medium angle, a similar evolution is observed around this point.
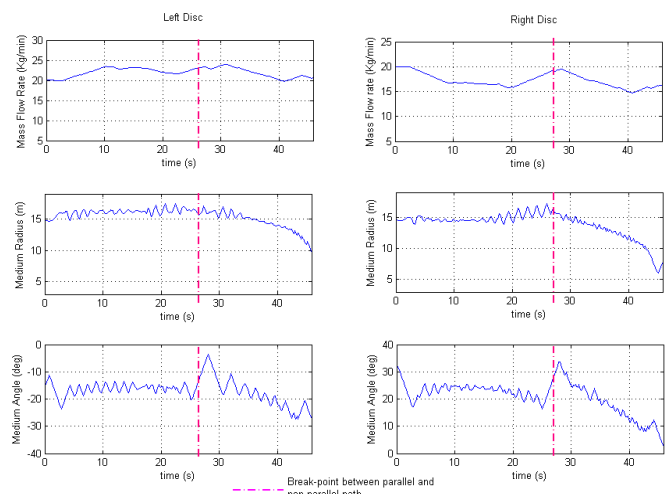

Figure 7: Optimal parameters for the first path

Figure 8 shows an increasing of all parameters for each disc. This phenomenon can be explained by the narrow pass occurring when the tractor comes in the main field body. The values reached after stabilization are close to the ones set by manufacturers with $24 \mathrm{~m}$ spaced parallel trajectories.Figure 9 exposes a simulation result in the main field body with the optimized parameters. As we can notice, application errors are reduced comparing to the ones exposed in Figure 6. Optimization leads to errors included between $-10 \%$ and $+6.3 \%$, which is very satisfying with regard to environmental and economic requirements.

\section{CONCLUSION}

A new method for optimization of fertilizer application by centrifugal spreaders has been presented. To
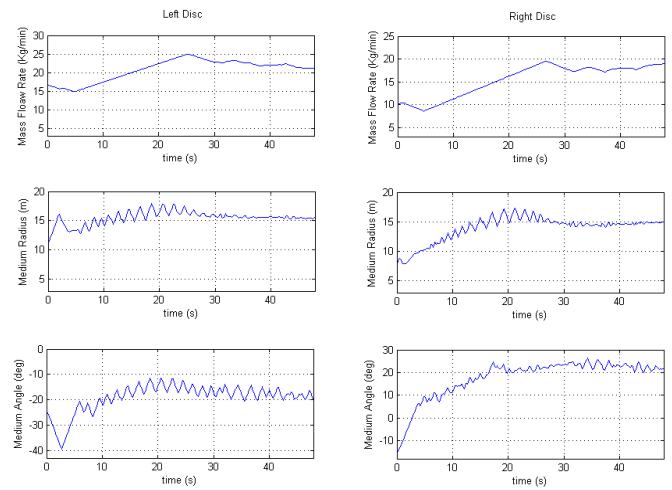

Figure 8: Optimal parameters for the second path

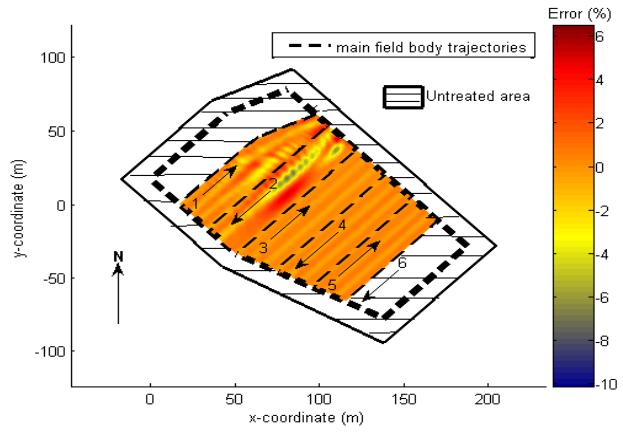

Figure 9: Application errors obtained after optimization

make sure that optimized parameters respect the actual process and can be used as reference variable for the future control of the machine, bounds constraints have been considered. Given the large scale problem resulting from the discretization, we have decomposed it and applied an augmented lagrangian algorithm with a l-bfgs technique. Optimal parameters enable to really limit application errors. Future studies are needed to optimize spreading process in boundaries zones. The computed variables for the whole field should then significantly enhance spreading accuracy.

\section{REFERENCES}

Bertsekas, D. P. (1982). Constrained Optimization and Lagrange Multipliers Methods. Academic Press, New York.

Bruxelles (2005). Mise en oeuvre de la directive 91/676/cee - pollution par les nitrates a partir de sources agricoles.

Byrd, R. H., Nocedal, J., and Schnabel, R. B. (1994). Representations of quasi-newton matrices and their use in limited memory methods. Mathematical Programming, 63:129-156.

Dillon, C. R., Shearer, S., Fulton, J., and Kanakasabai, M. (2003). Optimal path nutrient application using variable rate technology. In Proc. of the Four th European Conference on Precision Agriculture, pages 171-176.

Isherwood, K. F. (1998). Mineral Fertilizer Use and the Environment. IFA.

ISO (1985). ISO 5690/1 Equipment for distributing fertilizers - Test methods - Part 1: Full width fertilizer distributors. International Organization for Standardization, Geneve.

Olieslagers, R. (1997). Fertilizer distribution modelling for centrifugal spreader design. $\mathrm{PhD}$ thesis, K.U. Leuven.

Yule, I., Lawrence, H., and Murray, R. (2005). Performance of fertiliser spreading equipment for precision agriculture applications. In 1st International Symposium on Centrifugal Fertiliser Spreading, pages 10-18. 